\title{
Vasa praevia with meandering foetal vessels despite placental and umbilical cord insertion on the same side of the uterine wall: a case report
}

Tatsuro Horiuchi ${ }^{1}$, Hiroshi Sato $^{1}$, Katsunori Matsui ${ }^{1}$, Makiko Ikeda $^{1}$, Hajime Morishita ${ }^{1}$, and Masaya Hirose ${ }^{1}$

${ }^{1}$ Hyogo Prefectural Amagasaki General Medical Center

January 16, 2021

\begin{abstract}
A 38-year-old patient with gravida 2, para 1 was referred to our hospital for perinatal management. At 37 weeks gestation, we diagnosed vasa praevia with meandering foetal vessels. Emergent caesarean section was performed. Obstetricians should be familiar with vasa praevia with meandering foetal vessels despite its rare occurrence.
\end{abstract}

Vasa praevia with meandering foetal vessels despite placental and umbilical cord insertion on the same side of the uterine wall: a case report

\section{Authors}

Tatsuro Horiuchi, Hiroshi Sato, Katsunori Matsui,

Makiko Ikeda, Hajime Morishita, Masaya Hirose

\section{Affiliation}

Department of Obstetrics and Gynecology, Hyogo Prefectural Amagasaki General Medical Center, Amagasaki, Japan

\section{Corresponding Author}

Hiroshi Sato

Department of Obstetrics and Gynecology

Hyogo Prefectural Amagasaki General Medical Center

2-17-77 Higashinaniwa-cho Amagasaki, Hyogo Prefecture 660-8550, Japan

E-mail: shiro801219@yahoo.co.jp

Tel: +81-6-6480-7000 Fax: +81-6-6480-7001

Short running title: Vasa praevia with meandering vessels

Vasa praevia with meandering foetal vessels despite placental and umbilical cord insertion on the same side of the uterine wall: a case report

\section{Abstract}


A 38-year-old patient with gravida 2, para 1 was referred to our hospital for perinatal management. At 37 weeks gestation, we diagnosed vasa praevia with meandering foetal vessels. Emergent caesarean section was performed. Obstetricians should be familiar with vasa praevia with meandering foetal vessels despite its rare occurrence.

\section{Key Words}

Caesarean section, Placenta, Prenatal diagnosis, Ultrasound, Vasa praevia

\section{Key Clinical Message}

Vasa praevia with meandering foetal vessels is extremely rare. It is difficult to diagnose such vasa praevia prenatally. When caesarean section is performed, a change in the site of uterine incision may be required for safe delivery.

\section{Introduction}

Vasa praevia is defined as a condition in which the cord vessels are present in the membranes covering the internal cervical os. The prevalence of vasa praevia is approximately 1 in 2500 pregnancies. In 90-95\% of these cases, vasa praevia is associated with placenta praevia, a low-lying, bilobed, or succenturiate lobe placenta, or with velamentous umbilical cord insertion [1]. An abnormal position and/or morphology of the placenta should alert the physician to rule out vasa praevia. If the position and morphology of the placenta are normal on ultrasound examination, the possibility of foetal vessels running over the internal cervical os should be assessed for diagnosis of vasa praevia. In such cases, foetal vessels usually run over the internal cervical os and continue to the main placenta on the opposite uterine wall. If the placenta and umbilical cord insertion are on the same side of uterine wall, foetal vessels could potentially meander and run over the internal os. Such vasa praevia is extremely rare and difficult to diagnose prenatally. Here, we report a case of vasa praevia with meandering foetal vessels despite placenta and umbilical cord insertion on the same side of uterine wall.

\section{Case history / examination}

A 38-year-old patient, gravida 2, para 1 was referred to our hospital at 9 weeks of gestation following in vitro fertilization. Her past history included moyamoya disease. Delivery with epidural anaesthesia had been recommended by the neurosurgeon. Her family history noted that her father had died of moyamoya disease. At the second trimester screening, the placenta was attached on the anterior uterine wall. A low-lying placenta, succenturiate lobe, and/or multilobed placenta was not identified. The umbilical cord insertion was marginal. The site of the umbilical cord insertion was on the lower side of the placenta. Both the placental position and the site of umbilical cord insertion were above the upper end of the bladder. At that time, vasa praevia was not suspected. At 37 weeks of gestation, gestational hypertension occurred. Elective delivery of the pregnancy was required. Transvaginal ultrasound without colour doppler did not reveal an abnormal image between the foetal head and the internal cervical os (Figure 1A). However, ultrasound with colour doppler was performed in order to exclude for abnormal insertion of the umbilical cord before introduction of a transcervical balloon catheter for cervical ripening. A few foetal vessels were found between the foetal head and the internal cervical os by using colour and pulse doppler (Figure 1B, 1C).

\section{Differential diagnosis, investigations and treatment}

We diagnosed vasa praevia. Emergency caesarean section was performed.

We predicted that the foetal vessels were running on the anterior wall of the lower uterine segment. When the lower uterine segment was incised transversely, we were very careful not to injure the foetal vessels before delivery. Careful blunt entry using haemostats and fingertips was performed. The amniotic membrane was exposed without rupture of the membrane and injury to foetal vessels. Foetal vessels were found in the exposed amniotic membrane (Figure 2A). Because the vessels were running on both sides of membrane, the amniotic membrane was ruptured at the central part. A mature female baby was safely delivered. The neonate had a birth weight of $2890 \mathrm{~g}$ (appropriate for gestational age) and Apgar scores were 8 and 9 at 1 
and 5 minutes, respectively. We examined the intrauterine findings after delivery. As we diagnosed before the operation, a section of the foetal vessels was meandering and running over the internal cervical os (Figure $2 \mathrm{~B})$. Both the placental position and the site of the umbilical cord insertion were above the low transverse incision scar (Figure 2C).

\section{Outcome and follow-up}

The postoperative course was uneventful.

Written informed consent was obtained from the patient for publication of this case report and accompanying images.

\section{Discussion}

The purpose of this case report is to draw the attention of the obstetrical practitioner to the importance of detailed transvaginal ultrasound examination including colour doppler to prevent accidents caused by unusual vasa praevia rupture.

The guidelines of the International Society of Ultrasound and Gynecology (ISUOG) for the second trimester examination mention the position of the placenta [2]. If the lower placental edge reaches or overlaps the internal os, a follow-up examination in the third trimester is recommended. The guideline mentions that detailed examination for vasa praevia may not need to be performed if the position and morphology of the placenta are normal.

Prince reported a case of vasa praevia with anomalous umbilical cord formation [3]. In this case, vasa praevia was not diagnosed prenatally. Routine screening by transabdominal ultrasound in the second and third trimester showed an anterior placenta not covering the cervical os. Further examination for vasa praevia was not performed. Fortunately, vaginal delivery was possible without serious complications for the neonate. This case is similar to our case in having an unexpected course of foetal vessels which resulted in vasa praevia. In such cases, transvaginal ultrasound without colour doppler may not be able to detect vasa praevia. The accuracy of transvaginal ultrasound in the diagnosis of vasa praevia was previously reported to be high when performed in combination with colour Doppler [4]. Initially, obstetricians usually perform transvaginal ultrasound without colour doppler. If the placenta and/or umbilical cord vessels are not detected near the internal cervical os by ultrasound without colour doppler, further examinations including colour doppler may not be performed. In our case, ultrasound with colour doppler was performed in order to exclude for abnormal insertion of the umbilical cord before introduction of a transcervical foley catheter for cervical ripening. A few foetal vessels indicating vasa praevia were only detected after we performed ultrasound with colour doppler, an incidental and fortunate diagnosis.

After diagnosis of vasa praevia, caesarean section before rupture of foetal vessels is thought to be required for good prognosis of the neonate. If foetal vessels are running on the anterior lower segment of the uterus, obstetricians should be careful to avoid intraoperative rupture of foetal vessels. Aoki et al. reported a case of vasa praevia in which the cord vessels were running on the anterior lower uterine segment [5]. They made a horizontal incision on the uterine fundus to avoid rupture of the cord. In future pregnancies after horizontal incision on the uterine fundus, the risk of uterine rupture or placenta accreta are higher relative to that of an incision in the lower uterine segment. However, in their case they concluded that incision in the lower uterine segment would have had a higher risk of rupture of cord vessels and loss of massive foetal blood. We also predicted that the cord vessels were running on the anterior lower uterine segment in our case. Both the placental position and the site of umbilical cord insertion were above the low transverse incision scar. A few foetal vessels were meandering and running over the internal cervical os. We thought that safe delivery via low transverse incision was feasible by putting the foetal vessels at the lateral part of the incision scar. The precise diagnosis of vasa praevia and assessment of the course of the cord vessels is essential for a safe delivery. A change in the site of uterine incision may be required. If the selected incision is not a normal low transverse incision, an increased risk in future pregnancies should be considered.

Both the patient and her father had moyamoya disease. Although genetic testing was not performed, familial 
disease was suspected. RNF213, the gene related to angiogenesis and vascular inflammation was identified as a susceptibility gene for moyamoya disease [6]. Recent research on moyamoya disease has shown that RNF213 variants are associated not only with moyamoya disease but also with intracranial atherosclerosis and systemic vascular diseases, such as peripheral pulmonary artery stenosis and renal artery stenosis [7]. Vasculopathy of umbilical and placental vessels has not been reported. The main cause as to why vasa praevia occurred in our case is not clear. However, a specific gene such as RNF213 may be related to anomalous formation of umbilical cord vessels which cause vasa praevia.

\section{Acknowledgement}

None

\section{Conflict of interests}

All authors declare no conflict of interests.

\section{Reference}

1. Bronsteen R, Whitten A, Balasubramanian M, et al. Vasa previa: clinical presentations, outcomes, and implications for management. Obstet Gynecol. 2013; 122: 352-357

2. Salomon LJ, Alfirevic Z, Berghella V, et al. ; ISUOG Clinical Standards Committee. Practice guidelines for performance of the routine mid-trimester fetal ultrasound scan. Ultrasound Obstet Gynecol. 2011 ;37 :116-126. doi: 10.1002/uog.8831. PMID: 20842655

3. Prince GD. Unruptured vasa previa with anomalous umbilical cord formation: a case of postpartum physician tachycardia Can Fam Physician. 2013 ;59 :1076-1078. PMID: 24130283 PMCID: PMC3796974

4. Ruiter L, Kok N, Limpens J, et al. Systematic review of accuracy of ultrasound in the diagnosis of vasa previa. Ultrasound Obstet Gynecol. $2015 ; 45: 516$.

5. Aoki M, Obata S, Odagami M, Miyagi E, Aoki S. Prenatal diagnosis of vasa previa and the course of the cord vessels contribute to the safety of cesarean sections: A case report. Clin Case Rep. $2019 ; 7$ :2114-2117. doi: 10.1002/ccr3.2432. eCollection 2019 Nov. PMID: 31788261 PMCID: PMC6878040 DOI: $10.1002 / \mathrm{ccr} 3.2432$

6. Liu W, Morito D, Takashima S, Mineharu Y, et al. Identification of RNF213 as a susceptibility gene for moyamoya disease and its possible role in vascular development. PLoS One. $2011 ; 6$ :e22542. doi: 10.1371/journal.pone.0022542. Epub 2011 Jul 20. PMID: 21799892

7. Bang OY, Chung JW, Kim DH, et al. Moyamoya Disease and Spectrums of RNF213 Vasculopathy. Transl Stroke Res. 2020 ;11 :580-589. doi: 10.1007/s12975-019-00743-6. Epub 2019 Oct 24. PMID: 31650369

\section{Figure legend}

\section{Figure 1}

Transvaginal ultrasound without colour doppler did not reveal an abnormal image such as the placenta and vessels between the foetal head and internal cervical os (1A). A vessel was found between the foetal head and the internal cervical os by using colour doppler (1B). Using pulse doppler, the vessel showed foetal artery waveforms (1C).

\section{Figure 2}

Intraoperative findings before rupture of the amniotic membrane showed that foetal vessels were found in the exposed amniotic membrane (2A). A segment of the foetal vessels was meandering and running over the internal cervical os (2B). Both the placental position and the site of the umbilical cord insertion were above the low transverse incision scar (2C). In all photographs, the upper side of image was the side of the patient's head. 

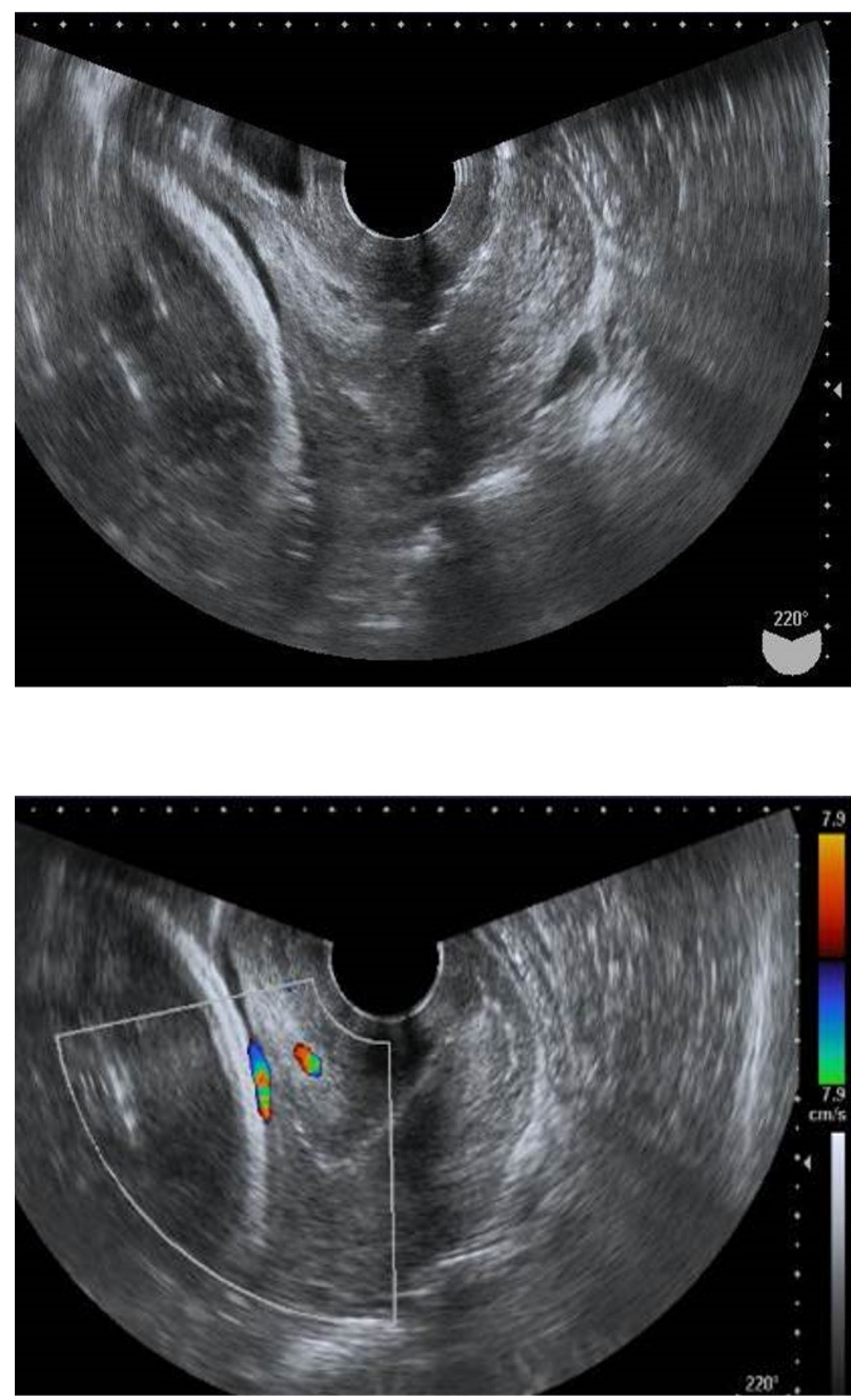

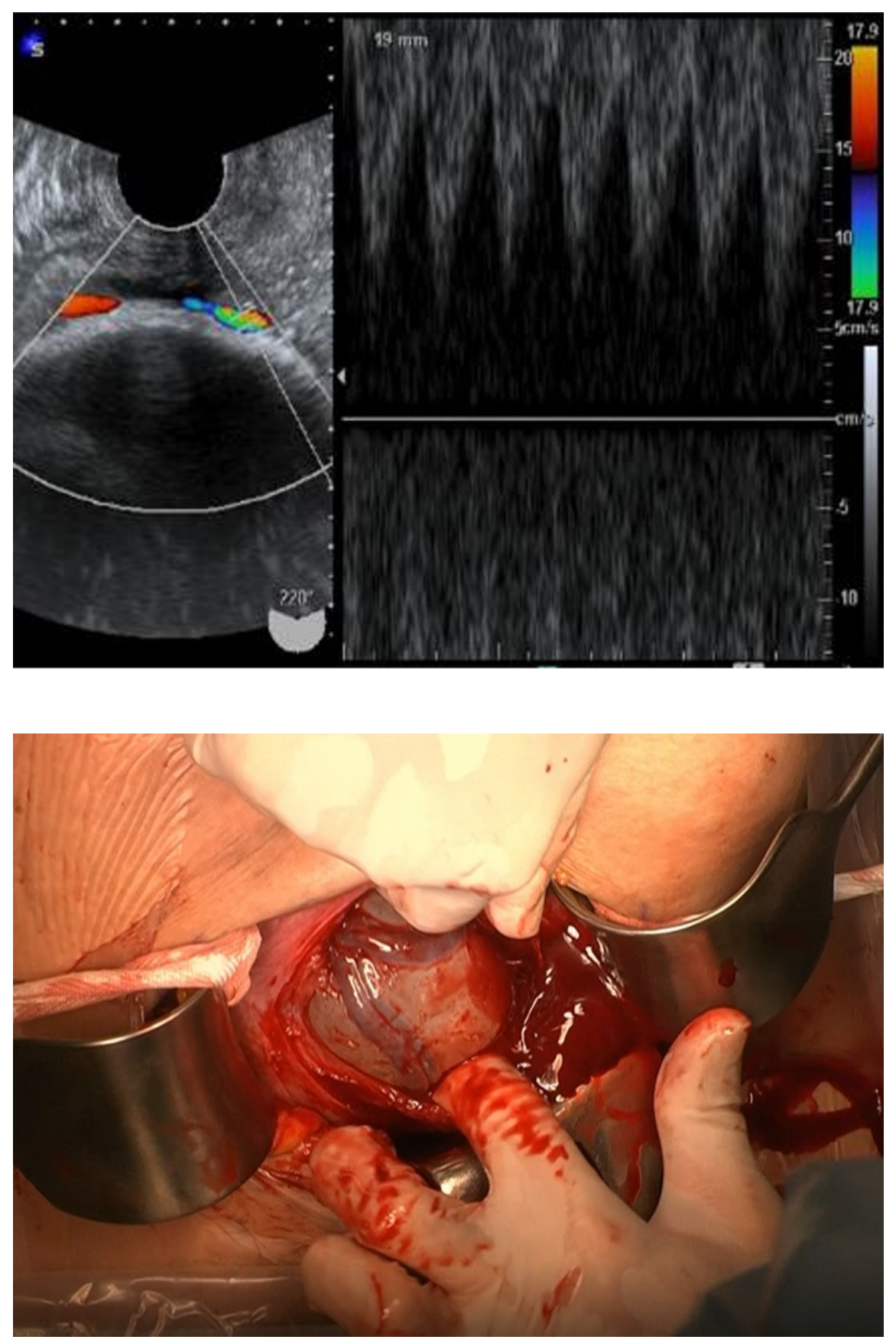

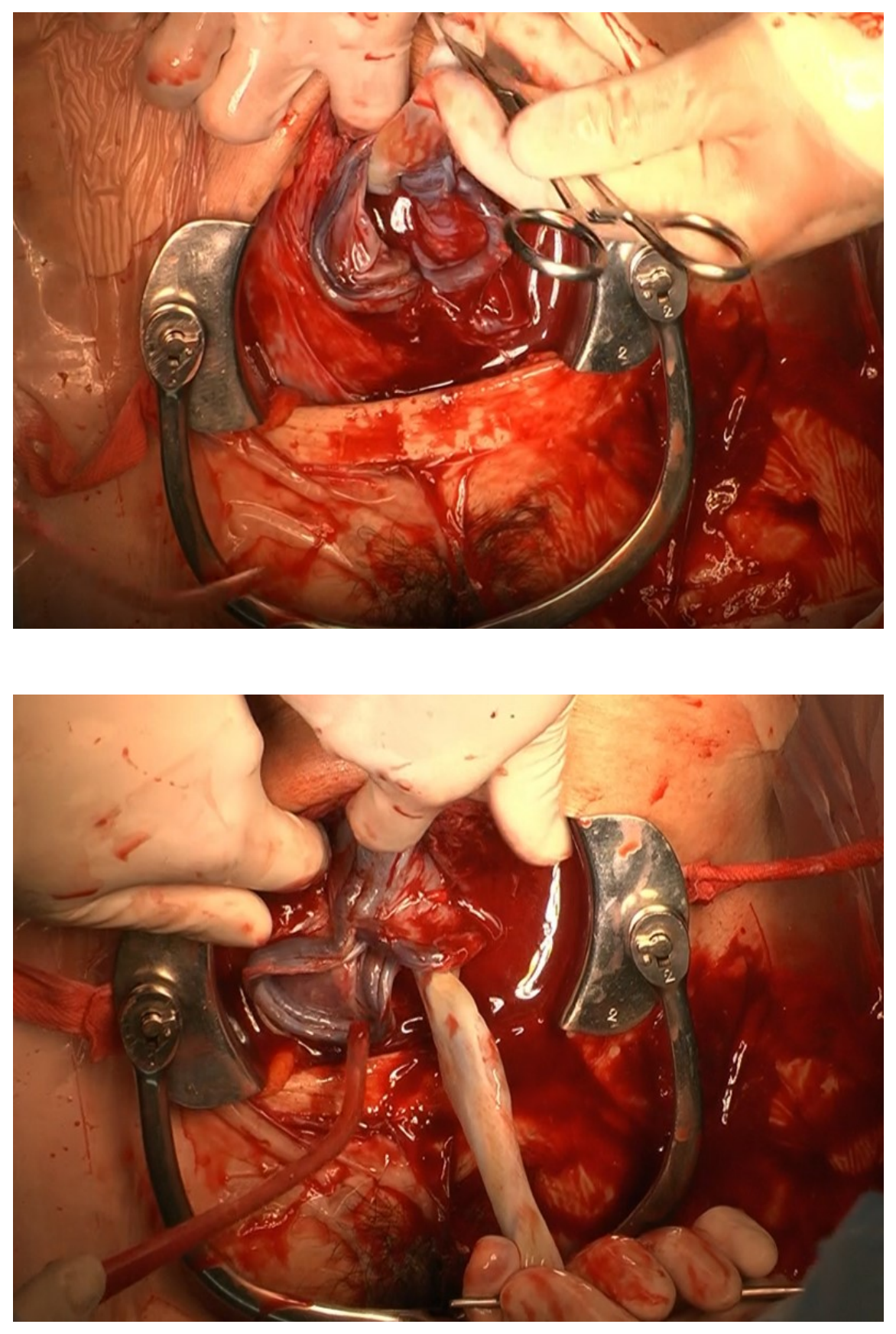